\title{
Infections Up to 76 Days After Stroke Increase Disability and Death
}

Learoyd, Annastazia E; Woodhouse, Lisa; Shaw, Laurence; Sprigg, Nikola; Bereczki, Daniel; Berge, Eivind; Caso, Valeria; Christensen, Hanne; Collins, Ronan; Czlonkowska, Anna; El Etribi, Anwar; Farr, Tracy D; Gommans, John; Laska, Ann-Charlotte; Ntaios, George; Ozturk, Serefnur; Pocock, Stuart J; Prasad, Kameshwar; Wardlaw, Joanna M; Fone, Kevin C; Bath, Philip M; Trueman, Rebecca C; ENOS Trial investigators

Published in:

Translational Stroke Research

DOI:

10.1007/s12975-017-0553-3

Publication date:

2017

Document version

Publisher's PDF, also known as Version of record

Document license:

CC BY

Citation for published version (APA):

Learoyd, A. E., Woodhouse, L., Shaw, L., Sprigg, N., Bereczki, D., Berge, E., Caso, V., Christensen, H., Collins, R., Czlonkowska, A., El Etribi, A., Farr, T. D., Gommans, J., Laska, A-C., Ntaios, G., Ozturk, S., Pocock, S. J., Prasad, K., Wardlaw, J. M., ... ENOS Trial investigators (2017). Infections Up to 76 Days After Stroke Increase Disability and Death. Translational Stroke Research, 8(6), 541-548. https://doi.org/10.1007/s12975-017-0553-3 


\title{
Infections Up to 76 Days After Stroke Increase Disability and Death
}

\author{
Annastazia E. Learoyd ${ }^{1} \cdot$ Lisa Woodhouse $^{2}$ - Laurence Shaw ${ }^{3}$ Nikola Sprigg ${ }^{2}$. \\ Daniel Bereczki $^{4} \cdot$ Eivind Berge $^{5} \cdot$ Valeria Caso $^{6} \cdot$ Hanne Christensen $^{7} \cdot$ Ronan Collins $^{8}$. \\ Anna Czlonkowska ${ }^{9}$ Anwar El Etribi ${ }^{10}$ - Tracy D. Farr ${ }^{1}$. John Gommans ${ }^{11}$. \\ Ann-Charlotte Laska ${ }^{12}$ - George Ntaios ${ }^{13}$ - Serefnur Ozturk ${ }^{14}$ - Stuart J. Pocock ${ }^{15}$. \\ Kameshwar Prasad $^{16}$ • Joanna M. Wardlaw ${ }^{17} \cdot$ Kevin C. Fone ${ }^{1}$. Philip M. Bath ${ }^{2}$. \\ Rebecca C. Trueman ${ }^{1} \cdot$ on behalf of the ENOS Trial investigators
}

Received: 4 April 2017 / Revised: 2 July 2017 / Accepted: 3 July 2017 / Published online: 27 July 2017

(C) The Author(s) 2017. This article is an open access publication

\begin{abstract}
Early infection after stroke is associated with a poor outcome. We aimed to determine whether delayed infections (up to 76 days post-stroke) are associated with poor outcome at 90 days. Data came from the international Efficacy of Nitric Oxide Stroke (ENOS, ISRCTN99414122) trial. Post hoc data on infections were obtained from serious adverse events reports between 1 and 76 days following stroke in this large cohort of patients. Regression models accounting for baseline covariates were used to analyse fatalities and functional
\end{abstract}

Members of the ENOS Trial investigators are listed in ENOS trial investigators. Lancet. 2015;385(9968):617-28

Electronic supplementary material The online version of this article (doi:10.1007/s12975-017-0553-3) contains supplementary material, which is available to authorized users.

Rebecca C. Trueman

Rebecca.Trueman@nottingham.ac.uk

1 School of Life Sciences, University of Nottingham, Nottingham, UK

2 Stroke Trials Unit Division of Clinical Neuroscience, School of Medicine, University of Nottingham, Nottingham, UK

3 School of Mathematical Sciences, University of Nottingham, Nottingham, UK

4 Department of Neurology, Semmelweis University, Budapest, Hungary

5 Department of Internal Medicine, Oslo University Hospital, Oslo, Norway

6 Stroke Unit, Santa Maria Hospital, University of Perugia, Perugia, Italy

7 Department of Neurology, Bispebjerg Hospital, Copenhagen, Denmark

8 Stroke Service, Adelaide and Meath Hospital, Tallaght, Ireland outcomes (modified Rankin Scale (mRS), Barthel Index, Euro-Qol-5D) at 90 days, in patients with infection compared to those without infection. Of 4011 patients, $242(6.0 \%)$ developed one or more serious infections. Infections were associated with an increased risk of death $(p<0.001)$ and an increased likelihood of dependency (measured by mRS) compared to those of all other patients $(p<0.001)$. This remained when only surviving patients were analysed, indicating that the worsening of functional outcome is not due to mortality
9 2nd Department of Neurology, Institute of Psychiatry and Neurology, Warsaw, Poland

10 Ainshams University, 7 Ahram Street, Heliopolis, Cairo, Egypt

11 Department of Medicine, Hawke's Bay Hospital, Hastings, New Zealand

12 Department of Clinical Sciences, Danderyd Hospital, Karolinska Institutet, Stockholm, Sweden

13 Department of Medicine, University of Thessaly, Larissa, Greece

14 Department of Neurology, Selcuk University Medical Faculty, Konya, Turkey

15 Department of Epidemiology and Population Health, London School of Hygiene and Tropical Medicine, London, UK

16 Department of Neurology, All India Institute of Medical Sciences, New Delhi, India

17 Division of Neuroimaging Sciences, Centre for Clinical Brain Sciences, Western General Hospital, Edinburgh, UK 
$(p<0.001)$. In addition, the timing of the infection after stroke did not alter its detrimental association with fatality $(p=0.14)$ or functional outcome $(p=0.47)$. In conclusion, severe poststroke infections, whether occurring early or late after stroke, are associated with an increased risk of death and poorer functional outcome, independent of differences in baseline characteristics or treatment. Not only are strategies needed for reducing the risk of infection immediately after stroke, but also during the first 3 months following a stroke. This study is registered: ISRCTN registry, number ISRCTN99414122, ClinicalTrials.gov Identifier, NCT00989716.

Keywords Stroke $\cdot$ Infection · Glyceryl trinitrate $\cdot$ Disability

\section{Introduction}

Approximately 15 million people in the world have a stroke each year [1]. Of these, $30.0 \%$ will develop infections in the first week post-stroke [2], which is associated with an increase in the likelihood of death $[3,4]$ and the extent of disability [5, 6]. These data, combined with the growing body of evidence from other neurological disorders, indicate that infection has a detrimental effect on the damaged brain $[7,8]$.

The risk of infection is increased after a stroke due to immunosuppression [9, 10], dysphagia and aspiration [11], cannulation/catherisation [12] and reduced mobility [13]. However, only a few studies have examined the effect of infections beyond the first 14 days after a stroke, and these showed that infections in the period from 14 up to 30 days post-stroke were associated with increased dependence on hospital discharge and increased mortality [14, 15].

The international multicentre Efficacy of Nitric Oxide in Stroke (ENOS) trial [16] collected data on all serious adverse events until 90 days post-randomisation, including common infections such as pneumonia and urinary tract infections [17]. Using the data collected in this trial, we aimed to perform a post hoc examination of the effect of infection up to 76 days post-stroke on survival and functional outcome and quality of life at 90 days post-randomisation.

\section{Methods \\ ENOS Trial}

The ENOS was an international multicentre trial of transdermal glyceryl trinitrate (GTN) or no GTN [16] in patients with acute ischaemic stroke and high blood pressure. Those taking antihypertensive medication were also randomised to continue or temporarily stop their medication in a partial factorial design. Patients were randomised within $48 \mathrm{~h}$ of stroke onset and treated for 7 days. This study is registered: ISRCTN registry, number ISRCTN99414122, ClinicalTrials.gov Identifier, NCT00989716.

Data collection for the ENOS was performed using a secure web interface and included the collection of baseline demographic (age, sex) and clinical variables (comorbidities, stroke type, subtype and severity [Scandinavian Stroke Scale, SSS]). Baseline data and main results have been reported previously $[16,18]$.

\section{Target Population for the Present Analysis}

In addition to the ENOS exclusion criteria [16, 19], the following patients were excluded (Fig. 1): those with a previous stroke (to reduce chance of patients having previous disability/ dependency), those with a non-stroke diagnosis and patients with serious adverse events (SAEs) reported after 76 days (in an attempt to prevent SAEs directly affecting outcome at 90 days). SAEs were defined as those events that were fatal, caused disability, were life-threatening, led to hospital admission, prolonged discharge in a hospitalised patient or were associated with birth defects in a child born to a trial

Total Patients: 4011

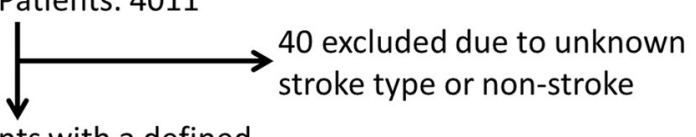

Patients with a defined

stroke (ischaemic or

haemorrhagic): 3971

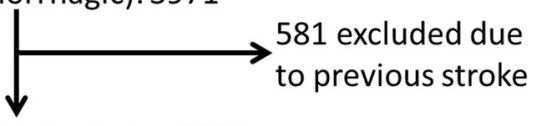

First Stroke Only: 3390

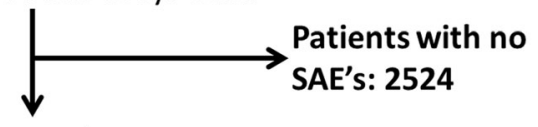

Patients who

developed SAEs: 866

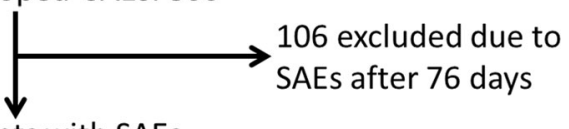

Patients with SAEs

between 1 and 76

days: 760

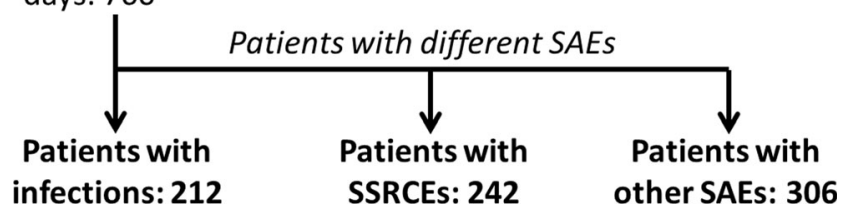

Fig. 1 Criteria used for selection of patients for analysis of the effects of infection. Patients were excluded due to lack of defined stroke type, previous stroke or serious adverse events (SAEs) after 76 days. Remaining patients were grouped based on the absence of SAEs or the presence of infections, secondary stroke-related cerebral events (SSRCEs) or other SAEs. Patients with infections were compared to all three of the other patient groups (individually or combined (termed: all other patients)) to assess the effect of infection on case fatality and functional outcome 
participant (http://www.nottingham.ac.uk/ nszwww/enos/ enosprotocolv15.pdf). SAEs that arose at any time point from the randomisation to final follow-up at day 90 were adjudicated blinded to treatment assignment by Daniel Bereczki and Nikola Sprigg.

Patients included in the analysis were grouped based on the presence of different types of SAEs. SAEs were categorised as infection, secondary stroke-related cerebral event (SSRCE) or other SAE. SSRCE was defined as cerebral oedema, complication or extension of initial stroke, haemorrhagic transformation, intracerebral/extracerebral/intracranial bleeds, transient ischaemic attack or recurrent stroke. These are known to worsen the outcomes being measured. Other SAEs included any other event ranging from falls to cardiac events (Supplementary Table I). Patients with no reported SAEs but who had died by 90 days (as determined by the outcome measures) were also placed in the other SAE category. Patients with multiple SAEs were categorised as SSRCEs (if they had at least one SSRCE) or infection (if they had no SSRCEs, but had infection). Patients with infection were also categorised by their time of presentation after randomisation: within 7 days (early), 8-28 days (intermediate) and 2976 days (late). This was to allow the effect of infections acquired at different time points to be delineated.

\section{Outcome Measures}

The outcome measures were case fatalities (by 90 days), functional/vital status as measured by the modified Rankin Scale (mRS, primary outcome) and activities of daily living (Barthel Index and Euro-QoL-5D Health Utility State [HUS]). The telephone-Mini Mental State Examination (t-MMSE), modified Zung Depression Scale (ZDS), modified Telephone Interview for Cognitive Status (TICS-M), EuroQol-Visual Analogue Scale (EQ-VAS) and verbal fluency (animals named in $1 \mathrm{~min}$ ) were also assessed in the ENOS trial but not used in the analysis due to insufficient numbers of responses in the subgroups $(<20)$. All functional measures were determined by telephone at 90 days by an assessor blinded to treatment and other clinical details.

\section{Statistics}

All analyses were performed using SPSS, version 22. Data are number (\%), mean (standard deviation, SD) or median (interquartile range, IQR). Significance is taken at $p<0.05$. Adjustments for multiplicity of testing were not performed.

Case fatalities in patients with infections were compared to fatalities in all other patients, or to patients with either SSRCEs or other SAEs using Cox regression with infection, SSRCEs and other SAEs as time-updated covariates. This second analysis allowed patients with infection to be compared to other patient groups of a similar size whom also experienced serious adverse events. To control for factors which are known to alter outcome, other covariates were also included in these analyses: age, sex, hypertension, diabetes, use of alteplase, stroke type and severity at baseline, and the ENOS treatment groups. An additional model (analysing just patients with infection) was used to assess the effect of time of infection on fatality across the patient group.

Functional outcome (mRS, Barthel Index and HUS) in patients with infection and all other patients (without SAEs, with SSRCEs or with other SAEs) was analysed using ordered probit regression. The covariates described above were included in the analysis. Ordered probit regression was repeated using only patients who survived to 90 days in order to separate the effect of infection on functional outcome from that on mortality, as the functional outcome measures included death in the scoring system. To assess if the time that infection developed influenced outcome within the surviving population, regressions compared functional outcome in patients acquiring infections at each time points (1-7, 8-28 and 2976 days post-stroke) to functional outcome in all other patients. An additional model (analysing just patients with infection) was used to assess the effect of time of infection on dependency across the patient group. To control further for baseline differences and confirm the findings of regression analysis, patients with infections were paired with those that had no SAEs. These paired groups were compared using ordered probit regression without covariates (see Supplemental Table II and Figure II for full details).

\section{Results}

\section{Participants and SAE Occurrence}

Of the 4011 patients enrolled into the ENOS trial, 703 were excluded from this analysis. Of the remaining patients, 760 developed SAEs with 1091 SAEs reported in total. Two hundred ninety-three $(26.9 \%)$ of these SAEs were infections, which occurred in 242 patients. Thirty of these patients were removed from the infection group due to the additional presence of a SSRCE. Out of the 293 reported infections, 192 $(65.5 \%)$ were pneumonia and $57(19.5 \%)$ were urinary tract infections. Of the remaining SAEs reported, 260 (23.8\%) were SSRCEs and 538 (49.3\%) were other SAEs occurring in 242 and 306 patients, respectively. The characteristics of patients with infections, SSRCEs, other SAEs or no SAEs are shown in Table 1.

The incidence rate of infections, SSRCEs and other SAEs followed a non-linear pattern with more SAEs reported shortly after stroke onset. The median number of days between stroke onset and SAE occurrence was 4 (2-12) days for SSRCEs, 11 (3-32) days for infections and 14 (4-33) days for other SAEs. 
Table 1 Baseline characteristics of all patients in the ENOS trial and groups of patients used in this study

\begin{tabular}{llllll}
\hline Variable & All patients & Patients with: & & \\
\cline { 3 - 6 } & & Infections & SSRCEs & Other SAEs & No SAEs \\
\hline No. of patients & 4011 & 212 & 242 & 306 & 2524 \\
Age (years) & $70.3(12.2)$ & $77.8(9.9)$ & $72.5(12.7)$ & $72.7(11.1)$ & $68.7(12.1)$ \\
Sex, male & $2297(57.3 \%)$ & $118(55.7 \%)$ & $119(49.2 \%)$ & $157(51.3 \%)$ & $1486(58.9 \%)$ \\
Risk factors & & & & & \\
$\quad$ Hypertension & $2607(65.0 \%)$ & $134(63.2 \%)$ & $165(68.2 \%)$ & $195(63.7 \%)$ & $1549(61.4 \%)$ \\
$\quad$ Diabetes & $699(17.4 \%)$ & $35(16.5 \%)$ & $48(19.8 \%)$ & $45(14.7 \%)$ & $396(15.7 \%)$ \\
$\quad$ Thrombolysis & $425(10.6 \%)$ & $27(12.7 \%)$ & $32(13.2 \%)$ & $45(14.7 \%)$ & $252(10.0 \%)$ \\
Stroke type & & & & & $2097(83.1 \%)$ \\
Ischaemic & $3342(83.3 \%)$ & $181(85.4 \%)$ & $197(81.4 \%)$ & $275(89.9 \%)$ & $209(16.9 \%)$ \\
$\quad$ Haemorrhagic & $629(15.7 \%)$ & $31(14.6 \%)$ & $45(18.6 \%)$ & $31(10.1 \%)$ & $427)$ \\
Pre-morbid mRS & $0(0-1)$ & $0(0-1)$ & $0(0-0)$ & $0(0-0)$ & $0(0-0)$ \\
SSS score $(/ 58)$ & $36(24-44)$ & $22(12-30)$ & $26(14-39)$ & $32(21-41)$ & $38(28-46)$ \\
GTN treatment & $2000(49.9 \%)$ & $108(50.9 \%)$ & $135(55.8 \%)$ & $167(54.6 \%)$ & $1230(48.7 \%)$ \\
Time to treatment & $26.0(12.9)$ & $27.7(12.6)$ & $23.7(12.8)$ & $25.6(14.2)$ & $26.2(12.7)$ \\
Developed SAEs & $1057(26.4 \%)$ & & & & \\
Death by 90 days & $494(12.3 \%)$ & $113(53.3 \%)$ & $125(51.9 \%)$ & $91(29.7 \%)$ & $0(0 \%)$ \\
\hline
\end{tabular}

Data is described as number (\% of patients in group) for categorical data, mean (SD) for continuous data or median (IQR) for ordinal data

SSRCEs secondary stroke-related cerebral events, SAEs serious adverse events, SSS Scandinavian Stroke Scale, GTN glyceryl trinitrate

\section{Case Fatality Rate in Patients with Infections, SSRCEs or Other SAEs}

Infections significantly increased the likelihood of fatality (Fig. 2a; hazard ratio $[\mathrm{HR}]=6.47,95 \%$ confidence interval $[\mathrm{CI}]=5.01-8.35, p<0.001)$ compared to all patients, irrespective of other covariates. When compared to SSRCEs or other SAEs (Fig. 2b), both infections (HR $=1.94,95 \%$ $\mathrm{CI}=1.50-2.51, p<0.001)$ and SSRCEs $(\mathrm{HR}=2.02,95 \%$ $\mathrm{CI}=1.53-2.68, p<0.001)$ increased the likelihood of fatality. The occurrence of other SAEs did not affect fatality rate $(\mathrm{HR}=0.83,95 \% \mathrm{CI}=0.62-1.10, p=0.19)$. Analysis of the infection group alone showed that the timing of infection did not affect fatality $(\mathrm{HR}=0.99,95 \% \mathrm{CI}=0.99-1.00, p=0.14)$, indicating that late infections were as likely to cause death as those presenting in the days after stroke. In addition, $58.4 \%$ of patients destined to die after their infection did so in the first 2 days (Supplementary Figure I).

\section{Functional Outcome in Patients with Infection}

Infections significantly increased the likelihood of dependency as measured by the mRS (Fig. 3a; odds ratio [OR] $=2.90$, $95 \% \mathrm{CI}=2.46-3.42, p<0.001)$ compared to that of all other patients irrespective of other covariates. This finding was also present for the Barthel Index (Table 2, OR $=0.34,95 \%$
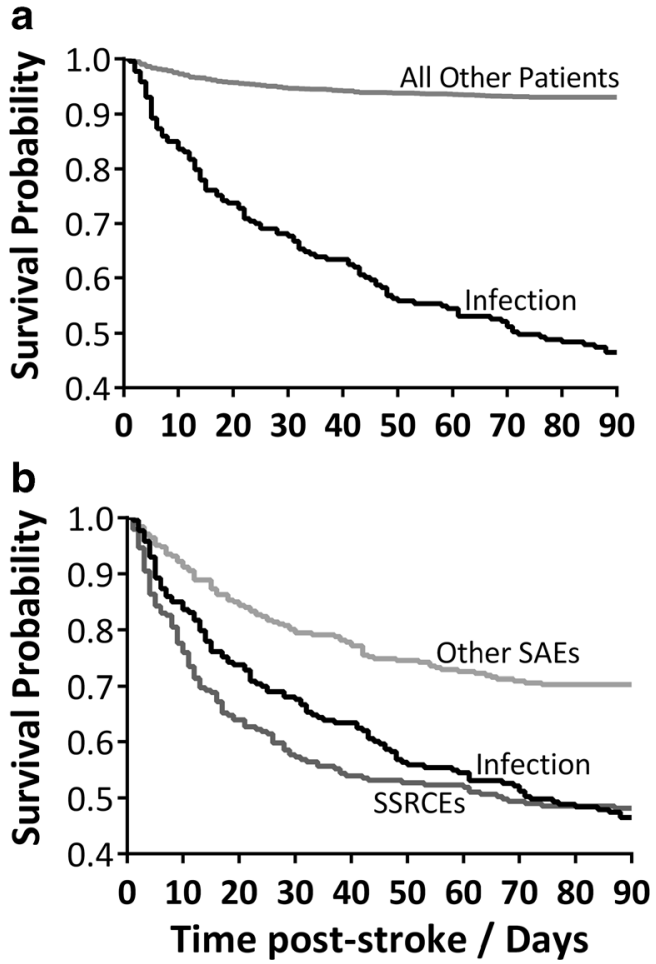

Fig. 2 Descriptive Kaplan-Meier graphs for patients developing infections between 1 and 76 days post-stroke and other groups of patients. a Patients with infections $(n=212)$ in comparison to all other patients (after exclusions, $n=3072$ ). b Patients with infections in comparison to those with secondary stroke-related cerebral events (SSRCEs, $n=242$ ) or with other serious adverse events (SAEs, $n=306$ ) 

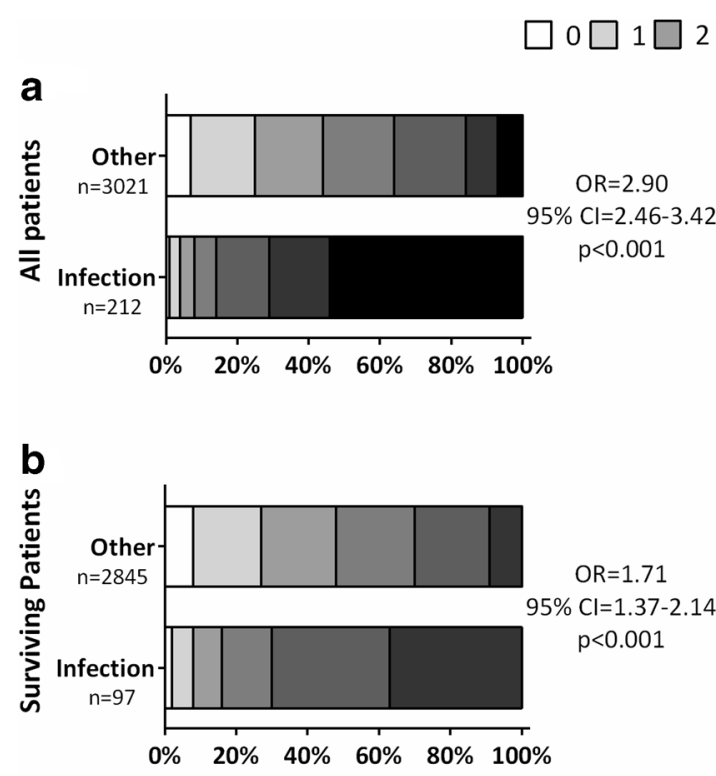

\section{$3 \square 4 \square 5 \square 6$ \\ c Individual Time Points}

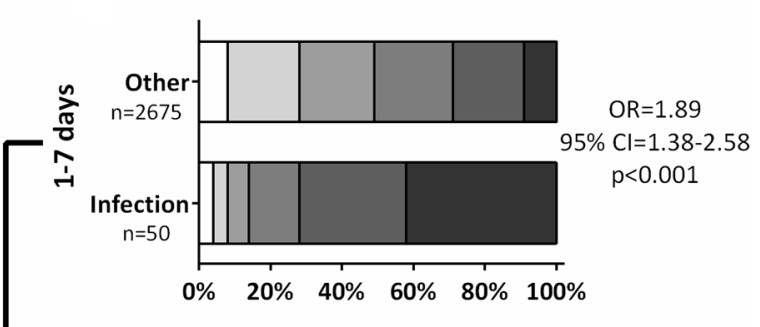

d
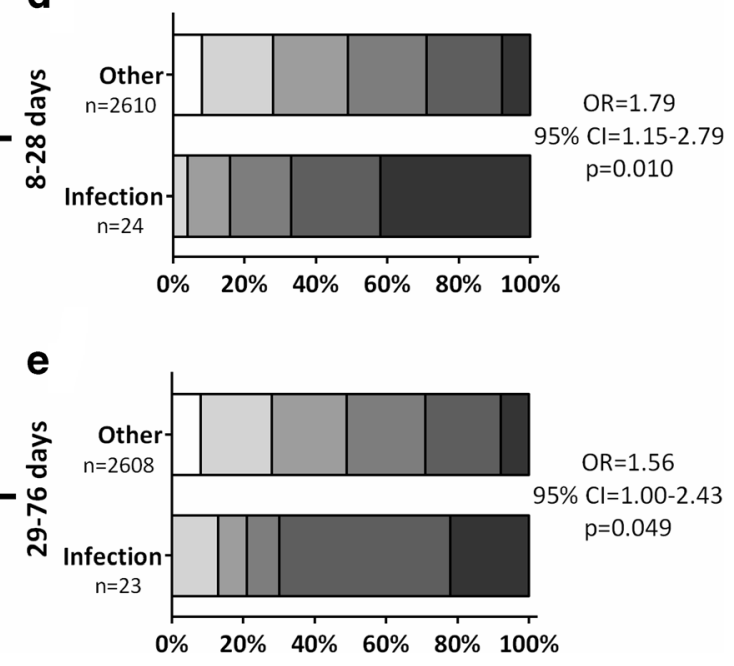

Fig. 3 Distributions in modified Rankin Scale scores at day 90 for stroke patients with infections and all other patients. a All patients with serious adverse events (SAEs) between 1 and 76 days post-stroke and patients without SAEs. b Patients who survived to 90 days only. c Surviving patients were split into patients who acquired infections or other SAEs

$\mathrm{CI}=0.28-0.40, p<0.001)$ and the HUS $(\mathrm{OR}=0.62,95 \%$ $\mathrm{CI}=0.53-0.72, p<0.001)$.

To establish that the reduction in functional outcome was not simply due to the increased mortality in the infection group, as the functional outcome measures include death as a score, the analysis was repeated using only patients who survived until 90 days. Patients who had infections continued

between 1-7, 8-28 and 29-76 days post-stroke. The infection group had higher Rankin Scores even when non-surviving patients were removed, indicating more dependency. This was maintained when patients were split into groups based on time of infection occurrence. $O R$ odds ratio, CI confidence interval

to exhibit a significantly worse functional outcome overall, as measured by the $\mathrm{mRS}$ (Fig. 3, OR $=1.71,95 \% \mathrm{CI}=1.37$ $2.14, p<0.001)$, than patients with no SAE. This difference was evident for infections between 1 and 7 days post-stroke $(\mathrm{OR}=1.89,95 \% \mathrm{CI}=1.38-2.58, p<0.001), 8-28$ days poststroke $(\mathrm{OR}=1.79,95 \% \mathrm{CI}=1.15-2.80, p=0.010)$ and 29 76 days post-stroke $(\mathrm{OR}=1.56,95 \% \mathrm{CI}=1.00-2.43$,

Table 2 Median scores (IQR) for Barthel Index and Health Utility State (HUS) in the comparison of stroke patients with infection and all other patients at defined time points

\begin{tabular}{lllll}
\hline Group & & $n$ & Barthel & HUS \\
\hline All patients & Infection & $209-212$ & $-5(-5 \text { to } 30)^{*}$ & $0.00(0.00-0.03)^{*}$ \\
& Other patients & $2995-3009$ & $85(50$ to 100$)$ & $0.64(0.19-0.81)$ \\
Surviving patients & Infection & $94-97$ & $35(8 \text { to } 75)^{*}$ & $0.03(-0.10 \text { to } 0.59)^{*}$ \\
& Other patients & $2798-2812$ & $90(60$ to 100$)$ & $0.66(0.26$ to 0.81$)$ \\
Between 1 and 7 days & Infection & $48-50$ & $30(5 \text { to } 71)^{*}$ & $0.03(-0.09 \text { to } 0.59)^{*}$ \\
& Other patients & $2631-2645$ & $90(65$ to 100$)$ & $0.69(0.26$ to 0.85$)$ \\
Between 8 and 28 days & Infection & $23-24$ & $43(6 \text { to } 83)^{*}$ & $0.03(-0.10 \text { to } 0.59)^{*}$ \\
& Other patients & $2570-2582$ & $90(65$ to 100$)$ & $0.69(0.26$ to 0.85$)$ \\
Between 29 and 76 days & Infection & 23 & $40(20 \text { to } 80)^{*}$ & $0.15(-0.09$ to 0.64$)$ \\
& Other patients & $2567-2579$ & $90(65$ to 100$)$ & $0.69(0.27$ to 0.85$)$ \\
\hline
\end{tabular}

$* p<0.05$ compared to other patients 
$p=0.049$ ). Analysis of surviving infection patients alone showed that the timing of infection did not affect functional outcome $(\mathrm{OR}=1.00,95 \% \mathrm{CI}=0.99-1.01, p=0.47)$. An additional analysis where patients with infection and patients without SAE were paired to control for variables such as age and stroke severity found similar results, whereby patients with infections consistently had reduced functional outcomes (Supplementary Figure II).

Infections at all three points were also associated with reduced functional outcome as measured by the Barthel Index (1-7 days: $\mathrm{OR}=0.54,95 \% \mathrm{CI}=0.40-0.72, p<0.001 ; 8-$ 28 days: $\mathrm{OR}=0.57,95 \% \mathrm{CI}=0.38-0.86, p=0.007 ; 29$ 76 days: $\mathrm{OR}=0.53,95 \% \mathrm{CI}=0.35-0.81, p=0.003)$. When measured using the HUS, patients with infections between 1 and 7 days post-stroke $(\mathrm{OR}=0.62,95 \% \mathrm{CI}=0.46-0.83$, $p=0.001)$ or $8-28$ days post-stroke $(\mathrm{OR}=0.66,95 \%$ $\mathrm{CI}=0.43-1.00, p=0.049$ ) had significantly worse functional outcome with a trend toward worse functional outcome in patients with infection between 29 and 76 days post-stroke $(\mathrm{OR}=0.67,95 \% \mathrm{CI}=0.45-1.02, p=0.063)$.

\section{Discussion}

The aim of this analysis was to determine whether severe infection at different time points post-stroke increases case fatality and dependency at 90 days post-stroke. Our study indicates that infections, at all time points assessed between 1 and 76 days, are associated with a higher incidence of death or dependency at 90 days. The time of infection onset did not alter the effect on death or functional outcome, demonstrating that infections that develop weeks after a stroke are as detrimental as those occurring within the first 7 days.

This work supports the current literature showing the detrimental effects of infection [3-6] and of systemic inflammation [20]. The likelihood of fatality after infection in these stroke patients is much higher than that seen in other patient populations with pneumonia [21,22], comparable to that after SSRCEs. In addition, these infections are associated with poor post-stroke recovery (independent to causing fatality) at much later time points than those which are established in the literature $[14,15,23]$. This highlights the need for long-term consideration of infections in patient care with measures to allow early identification and treatment. Unfortunately, prophylactic antibiotics do not, in themselves, appear to reduce rates of pneumonia infection, or improve functional outcome [24, 25]. Instead, measures to reduce aspiration such as awareness, diagnosis and management of dysphagia [26] or antiemetic treatment to reduce vomiting/regurgitation [27] are likely be more effective with ongoing trials hoping to address this [28]. However, no analyses of the use of preventive measures or the incidence of dysphagia were completed here due to lack of data.
Within this analysis, it is unclear how infections are contributing to functional deficits. Previous studies have shown that general medical complications after a stroke do influence rates of death and disability [23,29] and any adverse event is likely to have some effect on functional outcome. Time is critical when undergoing therapy, with rehabilitation being most effective in the first 30 days post-stroke [30]. However, the detrimental effect of later infections (from 29 to 76 days post-stroke) means that it is likely that infections are having a larger impact than just interfering with therapy. Mechanisms have been suggested for the effect of infection including an increased risk of further SAEs [31] and the development of an autoimmune response against the brain [32]. Inflammatory processes seem to be a likely contributor to the effect of infection. Pro-inflammatory T lymphocytes [32] and inflammatory markers such as IL-6 [20] have both been linked to poor outcome in stroke patients. Meanwhile, work in animal models has shown that activation of the peripheral immune system by lipopolysaccharide shortly after a stroke leads to increased microglia activation and neutrophil infiltration within the stroke infarct [33, 34] and worsens functional outcome [33, 35]. Finally, it has been shown in animal models of neurodegenerative diseases that peripheral inflammation leads to the reactivation of microglia within the brain [36]. It is possible that this is also occurring in stroke patients who get infections, particularly those with infections at later time points when the initial inflammatory responses to stroke onset are resolving.

\section{Limitations}

The strength of this study is that the data are based on a large multinational cohort of patients with high-fidelity data collection. However, there are several limitations to this study. First, this is a post hoc analysis of previously collected data from the ENOS database. During its conception, the ENOS investigators did not plan to investigate the effects of post-stroke infection and only collected most of their outcome measures for use as secondary outcomes [16]. This resulted in some gaps in data potentially reducing the numbers of patients in this analysis. Therefore, only those outcome measures with sufficient numbers of patients were used in this study.

Second, information on infection was not collected prospectively but rather using SAE reports which are known to show variability within and between sites and regions [37]. Therefore, it is possible that some SAEs were missed, particularly at later time points after hospital discharge. This is reflected in the incidence of SAEs and infections in this patient population. Only $23.4 \%$ of patients included in the analysis developed SAEs and only $27.9 \%$ of these patients had infections. In total, this means approximately $6.5 \%$ of patients developed infections, a lower frequency than that generally reported in the literature (30\% on average [17]). 
Third, the reliance on SAE reports to identify infections means that only serious infections were included in this analysis. Severity has been shown to have a role in determining how detrimental an infection is to post-stroke recovery $[2,23]$ and the fact that the effect of infection on fatality is higher than that of some previous reports supports this [3, 4]. Because of this, this dataset cannot be used to say that any infection is harmful to recovery regardless of time of infection. We can only be certain that severe infections are detrimental to functional outcome irrespective of time post-stroke.

Finally, as the patients with infection are on average older and have worse stroke severity, it is likely that their rate of death [38] and disability [39] would be higher without the additional complications of infection. It could be argued that these patients developed infections because they had worse functional outcome [40] rather than infection causing their decline, or that these patients may be given palliative care for their infections rather than aggressive treatment. Both of these factors would contribute to the high levels of death and disability. The influence of older age and stroke severity was accounted for by their inclusion as covariates within the analyses and by the use of patient pairing in an additional analysis of functional outcomes (Supplementary Table II and Figure II). Additionally, there is evidence at earlier time points post-stroke [14, 15], and from other diseases [7, 8], that infection accelerates the decline of neurological function, so the potential of infection to inhibit or limit recovery still needs to be taken seriously.

\section{Summary}

Infection at both early (1-7 days) and later ( $\leq 76$ days) time points post-stroke is detrimental to a person's functional recovery and survival from a stroke. Further work should establish whether it is possible to initiate prompt treatment of all infections to reduce their effects on mortality and functional outcome. In addition, work should be undertaken to investigate the mechanisms by which infections lead to increased deficits. Investigation into this area may be able to establish time periods of particular importance in post-stroke recovery, determine the types of infections which have the greatest influence or define the effect of post-stroke infection on a range of functional outcomes.

Acknowledgements We would like to thank the patients and investigators who took part in ENOS (as listed in the main publication [16]) and the National Institute for Health Research Stroke Research Network nurses/coordinators who contributed to recruitment into ENOS. The ENOS trial was primarily funded by the UK Medical Research Council (grant G0501797). Other funders who supported the trial were the Agency for Science, Technology, and Research (Singapore), BUPA Foundation (UK), Hypertension Trust (UK), Queen Elizabeth II Health Sciences Centre Research Fund (Canada), Reichstadt family (UK), and
The Stroke Association (UK, through its funding of the Division of Stroke, University of Nottingham, Nottingham, UK). AEL was funded by the Biotechnology and Biological Sciences Research Council. PB is the Chief Investigator of the ENOS and is the Stroke Association Professor of Stroke Medicine. The ENOS was sponsored by the University of Nottingham. Neither funder nor sponsor had any input into the study.

Author Contributions The majority of authors (NS, DB, EB, VC, HC, RC, AC, AEE, JG, ACL, GN, SO, SJP, KP, JMW, PMB) are members of the ENOS trial. AEL, PMB and RCT designed the study with critical input from SJP and TF. AEL completed the statistical analysis with assistance from LW, LS and SJP. AEL, PMB and RCT wrote the manuscript text with critical input from all the authors (NS, DB, EB, VC, HC, RC, AC, AEE, JG, ACL, GN, SO, SJP, KP, JMW, LW, LS, TF).

\section{Compliance with Ethical Standards}

Conflict of Interest The authors declare that they have no conflict of in terest.

Sources of Funding This work was supported by the Medical Research Council [grant number G0501797] and the Biotechnology and Biological Sciences Research Council [grant number BB/F016956/1]. Other funders who supported the ENOS trial were the Agency for Science, Technology, and Research (Singapore), BUPA Foundation (UK), Hypertension Trust (UK), Queen Elizabeth II Health Sciences Centre Research Fund (Canada), Reichstadt family (UK), and The Stroke Association (UK, through its funding of the Division of Stroke, University of Nottingham, Nottingham, UK). PB is the Chief Investigator of the ENOS and is the Stroke Association Professor of Stroke Medicine. ENOS was sponsored by the University of Nottingham. Neither funder nor sponsor had any input into the study.

Ethical Approval All procedures performed in studies involving human participants were in accordance with the ethical standards of the institutional and/or national research committee and with the 1964 Helsinki Declaration and its later amendments or comparable ethical standards.

Open Access This article is distributed under the terms of the Creative Commons Attribution 4.0 International License (http:// creativecommons.org/licenses/by/4.0/), which permits unrestricted use, distribution, and reproduction in any medium, provided you give appropriate credit to the original author(s) and the source, provide a link to the Creative Commons license, and indicate if changes were made.

\section{References}

1. Feigin VL, et al. Global and regional burden of stroke during 1990 2010: findings from the Global Burden of Disease Study 2010. Lancet. 2014;383(9913):245-54.

2. Aslanyan S, et al. Pneumonia and urinary tract infection after acute ischaemic stroke: a tertiary analysis of the GAIN International trial. Eur J Neurol. 2004;11(1):49-53.

3. Kwan J, Hand P. Infection after acute stroke is associated with poor short-term outcome. Acta Neurol Scand. 2007;115(5):331-8.

4. Heikinheimo T, et al. Preceding and poststroke infections in young adults with first-ever ischemic stroke: effect on short-term and longterm outcomes. Stroke. 2013;44(12):3331-7.

5. Wartenberg KE, et al. Infection after acute ischemic stroke: risk factors, biomarkers, and outcome. Stroke Res Treat. 2011;2011 
6. Hilker R, et al. Nosocomial pneumonia after acute stroke-implications for neurological intensive care medicine. Stroke. 2003;34(4):975-81.

7. Holmes C, et al. Systemic inflammation and disease progression in Alzheimer disease. Neurology. 2009;73(10):768-74.

8. Murta V, Ferrari CC. Influence of peripheral inflammation on the progression of multiple sclerosis: evidence from the clinic and experimental animal models. Mol Cell Neurosci. 2013;53:6-13.

9. Offner H, Vandenbark AA, Hurn PD. Effect of experimental stroke on peripheral immunity: CNS ischemia induces profound immunosuppression. Neuroscience. 2009;158(3):1098-111.

10. Czlonkowska A, Cyrta B, Korlak J. Immunological observations on patients with acute cerebral vascular disease. J Neurol Sci. 1979;43(3):455-64.

11. Titsworth WL, et al. Prospective quality initiative to maximize dysphagia screening reduces hospital-acquired pneumonia prevalence in patients with stroke. Stroke. 2013;44(11):3154-60.

12. Stott DJ, et al. Urinary tract infection after stroke. QJM. 2009;102(4):243-9.

13. Rogers MA, et al. Mobility and other predictors of hospitalization for urinary tract infection: a retrospective cohort study. BMC Geriatr. 2008;8:31.

14. Finlayson $\mathrm{O}$, et al. Risk factors, inpatient care, and outcomes of pneumonia after ischemic stroke. Neurology. 2011;77(14):1338-45.

15. Ulm L, et al. Impact of infections on long-term outcome after severe middle cerebral artery infarction. J Neurol Sci. 2012;319(1):15-7.

16. ENOS trial investigators. Efficacy of nitric oxide, with or without continuing antihypertensive treatment, for management of high blood pressure in acute stroke (ENOS): a partial-factorial randomised controlled trial. Lancet. 2015;385(9968):617-28.

17. Westendorp WF, et al. Post-stroke infection: a systematic review and meta-analysis. BMC Neurol. 2011;11(1):110.

18. ENOS Trial Investigators. Baseline characteristics of the 4011 patients recruited into the 'Efficacy of Nitric Oxide in Stroke' (ENOS) trial. Int J Stroke. 2014;9(6):711-20.

19. ENOS Trial Investigators. Glyceryl trinitrate vs. control, and continuing vs. stopping temporarily prior antihypertensive therapy, in acute stroke: rationale and design of the Efficacy of Nitric Oxide in Stroke (ENOS) trial (ISRCTN99414122). Int J Stroke. 2006;1(4): 245-9.

20. Whiteley W, et al. Inflammatory markers and poor outcome after stroke: a prospective cohort study and systematic review of interleukin-6. PLoS Med. 2009;6(9):e1000145.

21. Buzzo AR, et al. Morbidity and mortality of pneumonia in adults in six Latin American countries. Int J Infect Dis. 2013;17(9):e673-7.

22. Rothberg MB, et al. Outcomes of patients with healthcareassociated pneumonia: worse disease or sicker patients? Infect Control Hosp Epidemiol. 2014;35(S3):s107-15.

23. Hong KS, et al. Impact of neurological and medical complications on 3-month outcomes in acute ischaemic stroke. Eur J Neurol. 2008;15(12):1324-31.
24. Westendorp WF, et al. The Preventive Antibiotics in Stroke Study (PASS): a pragmatic randomised open-label masked endpoint clinical trial. Lancet. 2015;385(9977):1519-26.

25. Kalra L, et al. Prophylactic antibiotics after acute stroke for reducing pneumonia in patients with dysphagia (STROKE-INF): a prospective, cluster-randomised, open-label, masked endpoint, controlled clinical trial. Lancet. 2015;386(10006):1835-44.

26. Hinchey JA, et al. Formal dysphagia screening protocols prevent pneumonia. Stroke. 2005;36(9):1972-6.

27. Warusevitane A, et al. Safety and effect of metoclopramide to prevent pneumonia in patients with stroke fed via nasogastric tubes trial. Stroke. 2015;46(2):454-60.

28. Straatman J, et al. The PRECious trial PREdiction of Complications, a step-up approach, CRP first followed by CTscan imaging to ensure quality control after major abdominal surgery: study protocol for a stepped-wedge trial. Trials. 2015;16:382.

29. Johnston KC, et al. Medical and neurological complications of ischemic stroke: experience from the RANTTAS trial. RANTTAS Investigators. Stroke. 1998;29(2):447-53.

30. Salter K, et al. Impact of early vs delayed admission to rehabilitation on functional outcomes in persons with stroke. J Rehabil Med. 2006;38(2):113-7.

31. Ji R, et al. Interrelationship among common medical complications after acute stroke: pneumonia plays an important role. Stroke. 2013;44(12):3436-44.

32. Becker KJ, et al. Autoimmune responses to the brain after stroke are associated with worse outcome. Stroke. 2011;42(10):2763-9.

33. Langdon KD, MacLellan CL, Corbett D. Prolonged, 24-h delayed peripheral inflammation increases short- and long-term functional impairment and histopathological damage after focal ischemia in the rat. J Cereb Blood Flow Metab. 2010;30(8):1450-9.

34. Han D, et al. Clematichinenoside protects blood brain barrier against ischemic stroke superimposed on systemic inflammatory challenges through up-regulating A20. Brain Behav Immun. 2016;51:56-69.

35. Yousuf $\mathrm{S}$, et al. Post-stroke infections exacerbate ischemic brain injury in middle-aged rats: immunomodulation and neuroprotection by progesterone. Neuroscience. 2013;239:92-102.

36. Perry $\mathrm{VH}$, Holmes C. Microglial priming in neurodegenerative disease. Nat Rev Neurol. 2014;10(4):217-24.

37. Allen NB, et al. Regional variation in recommended treatments for ischemic stroke and TIA: get with the guidelines - stroke 20032010. Stroke. 2012;43(7):1858-64.

38. Bae HJ, et al. In-hospital medical complications and long-term mortality after ischemic stroke. Stroke. 2005;36(11):2441-5.

39. Knoflach M, et al. Functional recovery after ischemic stroke - a matter of age: data from the Austrian Stroke Unit Registry. Neurology. 2012;78(4):279-85.

40. Roth EJ, et al. Incidence of and risk factors for medical complications during stroke rehabilitation. Stroke. 2001;32(2):523-9. 